\title{
Computer Simulation of the Anisotropy of Fluorescence in Ring Molecular Systems: Tangential vs. Radial Dipole Arrangement
}

\author{
Pavel Heřman ${ }^{1}$, Ivan Barvík ${ }^{2}$, and David Zapletal ${ }^{1,3}$ \\ ${ }^{1}$ Department of Physics, Faculty of Education, University of Hradec Králové, \\ Rokitanského 62, CZ-500 03 Hradec Králové, Czech Republic \\ pavel.herman@uhk.cz \\ ${ }^{2}$ Institute of Physics of Charles University, Faculty of Mathematics and Physics, \\ CZ-12116 Prague, Czech Republic \\ ${ }^{3}$ Department of Mathematics, University of Pardubice, Studentská 95, \\ CZ-53210 Pardubice, Czech Republic
}

\begin{abstract}
Time dependence of the anisotropy of fluorescence in recently discovered cyclic antenna units of the BChl photosystem is modeled. Interaction with a bath and a static disorder here modeled as uncorrelated Gaussian disorder in the transfer integrals is taken into account. Parallel computer enviroment is used because one is forced to recalculate every physical quantity for several thousands of different realizations of disorder. Results for the ring LH4 with radial optical transition dipole arrangement are compared with those for the ring LH2 with the tangential one. The difference between LH2 and LH4 results for the static disorder in transfer integrals has an opposite sign in comparison with that one for the static disorder in local energies. Equivalent differences are shifted to a smaller times for the stronger interaction with a bath.
\end{abstract}

\section{Introduction}

The most common antenna complexes in purple bacteria are the light-harvesting complexes LH1 and LH2. Some bacteria express also other types of LH complexes such as the B800-820 LH3 complex in Rhodopseudomonas acidophila strain 7050 or the B800 LH4 complex in Rhodopseudomonas palustris 1. The general organization of the LH2 and LH4 complexes is the same: a ring-shaped structure is formed from cyclically repeated identical subunits. However, the symmetries of these rings are different: LH2 and LH3 are usually nonameric but LH4 is octameric. The other difference is the presence of four bacteriochlorophyll (BChl) molecules per repeating unit in LH4 rather than three ones found in LH2 and LH3. The most striking difference is the occurrence of an additional Bchl-a ring in the LH4 complex, the B800-2 ring, at a position approximately halfway between the densely packed B-a/B-b ring and the B800-1 ring that are both also present in LH2 11. In LH2, the B850 ring has nearly tangentially oriented Bchl-a pigments, whereas in LH4 the equivalent B-a/B-b pigments are organized in a more radial fashion.

M. Bubak et al. (Eds.): ICCS 2008, Part I, LNCS 5101, pp. 661670 2008.

(C) Springer-Verlag Berlin Heidelberg 2008 
We are therefore dealing with ring-shaped units with nonameric and octameric symmetry resembling those rings from antenna complexes LH2 and LH4 with a strong interaction $J$ (in the range $150-450 \mathrm{~cm}^{-1}$ ) between BChl molecules. Our theoretical approach therefore considers an extended Frenkel exciton states model.

Despite intensive study, the precise role of the protein moiety in governing the dynamics of the excited states is still under debate [2]. At room temperature the solvent and protein environment fluctuate with characteristic time scales ranging from femtoseconds to nanoseconds. The dynamical aspects of the system are reflected in the line shapes of electronic transitions. To fully characterize them and thereby the dynamics of the system, one needs to know not only the fluctuation amplitude (coupling strength) but also the time scale of each process involved. The observed linewidth reflects the combined influence of static disorder and exciton coupling to intermolecular, intramolecular, and solvent nuclear motions. The simplest approach is to decompose the line profile into homogeneous and inhomogeneous contributions of the dynamic and static disorder. Yet, a satisfactory understanding of the nature of static disorder in light-harvesting systems has not been reached. In the site excitation basis, static disorder can be present as in diagonal hamiltonian matrix elements as in off-diagonal ones.

Time-dependent experiments 34] led for the B850 ring in LH2 complex to conclusion that the elementary dynamics occurs on a time scale of about 100 fs 567 . For example, depolarization of fluorescence was studied already quite some time ago for a model of electronically coupled molecules [89. Rahman et al. [8] were the first who recognize the importance of the off-diagonal density matrix elements (coherences) 10 which can lead to an initial anisotropy larger than the incoherent theoretical limit of 0.4 . Already some time ago substantial relaxation on the time scale of 10-100 fs and an anomalously large initial anisotropy of 0.7 was observed by Nagarjan et al. 57. The high initial anisotropy was ascribed to a coherent excitation of a degenerate pair of states with allowed optical transitions and then relaxation to states at lower energies which have forbidden transitions. Nagarjan et al. 6] concluded, that the main features of the spectral relaxation and the decay of anisotropy are reproduced well by a model considering decay processes of electronic coherences within the manifold of the excitonic states and thermal equilibration among the excitonic states. In that contribution the exciton dynamics was not calculated explicitly.

In several steps [11/2 13|14|15 16 17] we have extended the former investigations by Kumble and Hochstrasser [18 and Nagarjan et al. 6] for LH2 rings. We added the effect of dynamic disorder by using a quantum master equation in the Markovian [1] and non-Markovian limits [1314]. We also investigated influence of four types of the uncorrelated static disorder (Gaussian disorder in local energies, transfer integrals, radial positions of BChls and angular positions of BChls) [12 15 16 17]. Influence of correlated static disorder, namely an elliptical deformation of the ring, has been also investigated [1].

Recently we have investigated the time dependence of the anisotropy of fluorescence for newly discovered type of the molecular ring, the LH4 ring with 
the uncorrelated static disorder in local energies [19]. Main goal of our present investigation is the comparison of the time dependence of the anisotropy of fluorescence after an impulsive excitation for two molecular rings: for molecular ring with tangentially arranged optical transition dipoles $r_{t}(t)$, like in LH2, as well as for the radially arranged one $r_{r}(t)$ like in LH4 [1]. We concentrate on the uncorrelated static disorder - Gaussian disorder in transfer integrals.

\section{$2 \quad$ Model}

In the following we assume that only one excitation is present on the ring after an impulsive excitation [18. The Hamiltonian of an exciton in the ideal ring coupled to a bath of harmonic oscillators reads

$$
\begin{gathered}
H^{0}=\sum_{m, n(m \neq n)} J_{m n} a_{m}^{\dagger} a_{n}+\sum_{q} \hbar \omega_{q} b_{q}^{\dagger} b_{q}+\frac{1}{\sqrt{N}} \sum_{m} \sum_{q} G_{q}^{m} \hbar \omega_{q} a_{m}^{\dagger} a_{m}\left(b_{q}^{\dagger}+b_{-q}\right)= \\
=H_{\mathrm{ex}}^{0}+H_{\mathrm{ph}}+H_{\mathrm{ex}-\mathrm{ph}}
\end{gathered}
$$

$H_{\mathrm{ex}}^{0}$ represents the single exciton, i.e. the system. The operator $a_{m}^{\dagger}\left(a_{m}\right)$ creates (annihilates) an exciton at site $m$. $J_{m n}$ (for $m \neq n$ ) is the so-called transfer integral between sites $m$ and $n . H_{\mathrm{ph}}$ describes the bath of phonons in the harmonic approximation. The phonon creation and annihilation operators are denoted by $b_{q}^{\dagger}$ and $b_{q}$, respectively. The last term in Eq. (10), $H_{\mathrm{ex}-\mathrm{ph}}$, represents the excitonbath interaction which is assumed to be site-diagonal and linear in the bath coordinates. The term $G_{q}^{m}$ denotes the exciton-phonon coupling constant.

Inside one ring the pure exciton Hamiltonian $H_{\mathrm{ex}}^{0}$ (Eq. (1)) can be diagonalized using the wave vector representation with corresponding delocalized "Bloch" states and energies. Considering homogeneous case with only nearest neighbour transfer matrix elements $J_{m n}=J_{12}\left(\delta_{m, n+1}+\delta_{m, n-1}\right)$ and using Fourier transformed excitonic operators (Bloch representation)

$$
a_{k}=\sum_{n} a_{n} e^{i k n}, \quad k=\frac{2 \pi}{N} l, \quad l=0, \pm 1, \ldots \pm N / 2,
$$

the simplest exciton Hamiltonian in $\boldsymbol{k}$ representation reads

$$
H_{\mathrm{ex}}^{0}=\sum_{k} E_{k} a_{k}^{\dagger} a_{k}, \quad \text { with } E_{k}=-2 J_{12} \cos k .
$$

In the local site basis influence of static disorder is modeled by a Gaussian distribution for the uncorrelated transfer integral fluctuations $\delta J_{n m}$ with a standard deviation $\Delta_{J}$

$$
H_{\mathrm{s}}=\sum_{m, n(m \neq n)} \delta J_{m n} a_{m}^{\dagger} a_{n} .
$$

We are using nearest neighbour approximation $J=J_{12}$. Hamiltonian of the static disorder adds to the Hamiltonian of the ideal ring

$$
H=H^{0}+H_{\mathrm{s}} \text {. }
$$


All of the $Q_{y}$ transition dipole moments of the chromophores (BChls B850) in a ring without static and dynamic disorder lie approximately in the plane of the ring and the entire dipole strength of the B850 band comes from a degenerate pair of orthogonally polarized transitions (at an energy slightly higher than the transition energy of the lowest exciton state (LH2), slightly lower than the one of the highest exciton state (LH4)).

The dipole strength $\boldsymbol{\mu}_{a}$ of eigenstate $|a\rangle$ of the ring with static disorder and the dipole strength $\boldsymbol{\mu}_{\alpha}$ of eigenstate $|\alpha\rangle$ of the ring without static disorder read

$$
\boldsymbol{\mu}_{a}=\sum_{n=1}^{N} c_{n}^{a} \boldsymbol{\mu}_{n}, \quad \boldsymbol{\mu}_{\alpha}=\sum_{n=1}^{N} c_{n}^{\alpha} \boldsymbol{\mu}_{n}
$$

where $c_{n}^{\alpha}$ and $c_{n}^{a}$ are the expansion coefficients of the eigenstates of the unperturbed ring and the disordered one in site representation, respectively. In the case of impulsive excitation the dipole strength is simply redistributed among the exciton levels due to disorder [18. Thus the impulsive excitation with a pulse of sufficiently wide spectral range will always prepare the same initial state, irrespective of the actual eigenstates of the real ring. After impulsive excitation with polarization $\boldsymbol{e}_{x}$ the excitonic density matrix $\rho[12$ is given by $[6]$

$$
\rho_{\alpha \beta}\left(t=0 ; \boldsymbol{e}_{x}\right)=\frac{1}{A}\left(\boldsymbol{e}_{x} \cdot \boldsymbol{\mu}_{\alpha}\right)\left(\boldsymbol{\mu}_{\beta} \cdot \boldsymbol{e}_{x}\right), \quad A=\sum_{\alpha}\left(\boldsymbol{e}_{x} \cdot \boldsymbol{\mu}_{\alpha}\right)\left(\boldsymbol{\mu}_{\alpha} \cdot \boldsymbol{e}_{x}\right) .
$$

The usual time-dependent anisotropy of fluorescence

$$
r(t)=\frac{\left\langle S_{x x}(t)\right\rangle-\left\langle S_{x y}(t)\right\rangle}{\left\langle S_{x x}(t)\right\rangle+2\left\langle S_{x y}(t)\right\rangle}, \quad S_{x y}(t)=\int P_{x y}(\omega, t) d \omega
$$

is determined from

$$
P_{x y}(\omega, t)=A \sum_{a} \sum_{a^{\prime}} \rho_{a a^{\prime}}(t)\left(\boldsymbol{\mu}_{a^{\prime}} \cdot \boldsymbol{e}_{y}\right)\left(\boldsymbol{e}_{y} \cdot \boldsymbol{\mu}_{a}\right)\left[\delta\left(\omega-\omega_{a^{\prime} 0}\right)+\delta\left(\omega-\omega_{a 0}\right)\right] .
$$

The brackets \langle\rangle in Eq. (77) denote the ensemble average and the orientational average over the sample.

The crucial quantity entering $r(t)$ in Eq. (7) is the exciton density matrix $\rho$. The dynamical equations for $\rho$ obtained by Cápek [20] read

$$
\frac{d}{d t} \rho_{m n}(t)=\sum_{p q} \mathrm{i}\left(\Omega_{m n, p q}+\delta \Omega_{m n, p q}(t)\right) \rho_{p q}(t) .
$$

In long time approximation coefficient $\delta \Omega(t \rightarrow \infty)$ becomes time independent.

All details of calculations leading to the time convolution-less dynamical equations for $\rho(t)$ are given elsewhere [14] and we shall not repeat them here. Obtaining of the full time dependence of $\delta \Omega(t)$ is not a simple task. We have succeeded to calculate microscopically full time dependence of $\delta \Omega(t)$ only for the simplest molecular model namely dimer 21 . In case of molecular ring we should resort 
to some simplification [14. In what follows we use Markovian version of Eq. (9) with a simple model for correlation functions $C_{m n}$ of the bath assuming that each site (i.e. each chromophore) has its own bath completely uncoupled from the baths of the other sites. Furthermore it is assumed that these baths have identical properties 322 . Then only one correlation function $C(\omega)$ of the bath is needed

$$
C_{m n}(\omega)=\delta_{m n} C(\omega)=\delta_{m n} 2 \pi\left[1+n_{B}(\omega)\right][J(\omega)-J(-\omega)]
$$

Here $J(\omega)$ is the spectral density of the bath 22] and $n_{B}(\omega)$ the Bose-Einstein distribution of phonons. The model of $J(\omega)$ often used in literature is

$$
J(\omega)=\Theta(\omega) j_{0} \frac{\omega^{2}}{2 \omega_{c}^{3}} \mathrm{e}^{-\omega / \omega_{c}}
$$

and has its maximum at $2 \omega_{c}$.

\section{Numerical Solution}

For the time propagation of the density matrix $\rho$ (Eq. 9) the short iterative Arnoldi method [23] as well as the standard Runge-Kutta scheme have been used. An advantage of the first one with respect to the second one is the low computational effort for moderate accuracy [24. Furthermore, the expansion coefficients are adapted at each time to a fixed time step with a prespecified tolerance in contrast to the Runge-Kutta scheme in which the time step is adapted. An uniform time grid is important for averaging of various realizations at the same time points without interpolation. The realization averaging and the orientational averaging can easily be parallelized by means of Message passing interface (MPI). Some computations were performed on a PC cluster. So instead of running about 10000 realizations on one node, 312 realizations can be calculated on each of the 32 nodes (or 52 realizations on each of 192 nodes).

Results of our simulations are presented graphically in the next section. We use dimensionless energies normalized to the transfer integral $J_{12}=J$ and the renormalized time $\tau$. To convert $\tau$ into seconds one has to divide $\tau$ by $2 \pi c J$ with $c$ being the speed of light in $\mathrm{cm} \mathrm{s}^{-1}$ and $J$ in $\mathrm{cm}^{-1}$. Estimation of $J$ varies between $250 \mathrm{~cm}^{-1}$ and $400 \mathrm{~cm}^{-1}$. Our time unit $(\tau=1)$ corresponds for these extreme values to 21.2 fs or 13.3 fs.

\section{Results}

Molecular ring is common shape of many antenna units in bacterial photosynthetic systems. They differ by number of BChl molecules, orientation of their optical transition dipoles, interchromophor distance, etc. We present graphically results of our modeling for time dependence of the fluorescence anisotropy in recently discovered cyclic antenna unit of the BChl photosystem, namely in LH4 


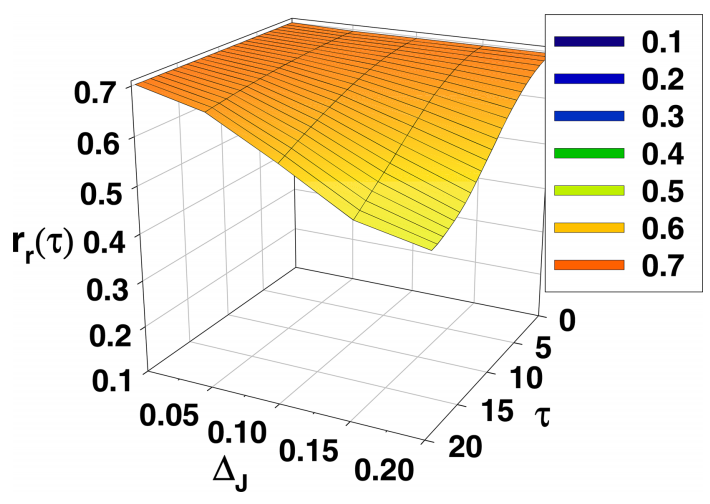

Fig. 1. The time and $\Delta_{J}$ dependence of the anisotropy of fluorescence $r_{r}$ of the LH4 (octameric) ring (without dynamic disorder)

with radial optical transition dipole arrangement. The transition dipole arrangement has a pronounced effect on the strength of the interaction $J$ between BChl molecules. The width of the exciton energy band of the ideal ring is two times larger for the tangential arrangement as in LH2 in comparison with radial arrangement in LH4. Also signs of $J$ are opposite in both configurations. Optically accessible exciton states in ideal rings are near the bottom (upper) edge of the exciton band in LH2 (LH4) respectively.

The time dependence of fluorescence anisotropy (Eq. (7)) has been calculated using dynamical equations for the exciton density matrix $\rho$ to express the time dependence of the optical properties of the ring units in the femtosecond time range. Details are the same as in Ref. 1415/16]. Substantial relaxation on the
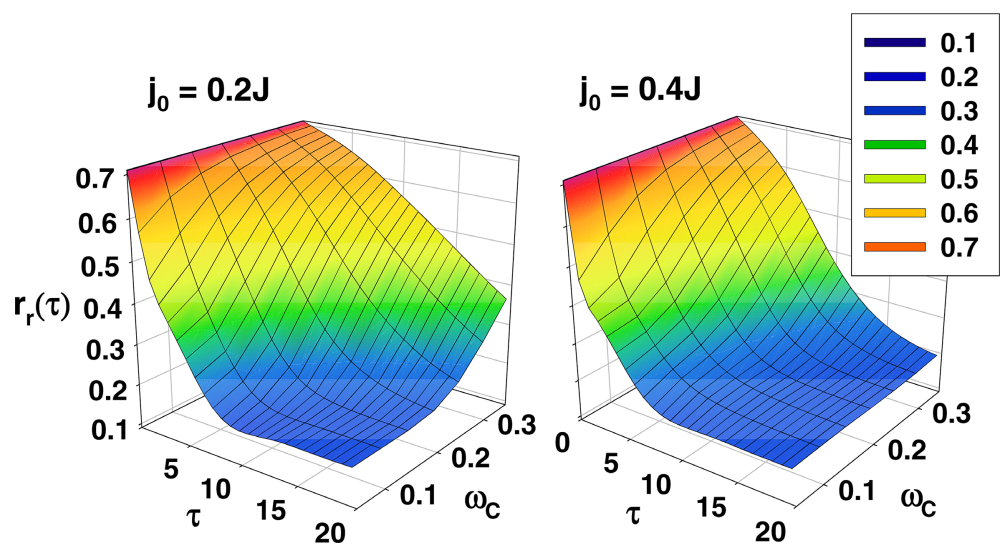

Fig. 2. The time and $\omega_{c}$ dependence of the anisotropy of fluorescence $r_{r}$ of the LH4 (octameric) ring. The dynamic disorder is included at $T=0.5 \mathrm{~J}\left(j_{0}=0.2 \mathrm{~J}\right.$ - left, $j_{0}=0.4 J$ - right). 


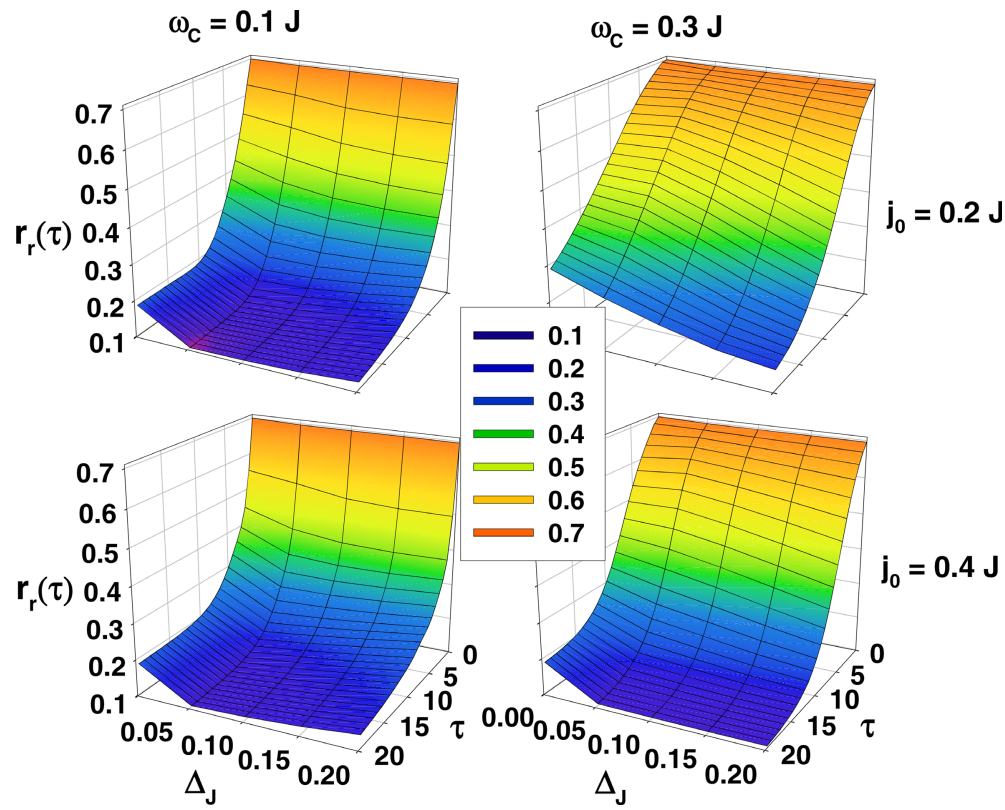

Fig. 3. The time and $\Delta_{J}$ dependence of the anisotropy of fluorescence $r_{r}$ of the LH4 (octameric) ring. The dynamic disorder is also included at $T=0.5 \mathrm{~J}\left(j_{0}=0.2 \mathrm{~J}\right.$ upper row, $j_{0}=0.4 \mathrm{~J}$ - lower row, $\omega_{c}=0.1 \mathrm{~J}$ - left column, $\omega_{c}=0.3 \mathrm{~J}$ - right column).

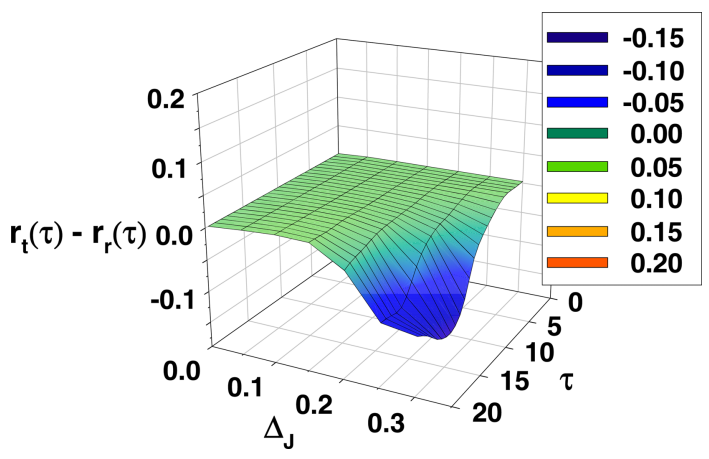

Fig. 4. The time and $\Delta_{J}$ dependence of the difference between anisotropy of fluorescence $r_{t}-r_{r}$ of the LH2 (nonameric) ring and the LH4 (octameric) one (without dynamic disorder)

time scale of 10-100 fs and an anomalously large initial anisotropy of 0.7 has been observed. Nagarjan et al. 6] suggested a model considering decay processes of electronic coherences within the manifold of the exciton states and thermal equilibration among the excitonic states. He supposed (without explicit calculation of the exciton dynamics) that this model reproduces well main features of the spectral relaxation and the decay of anisotropy in cyclic molecular units. 

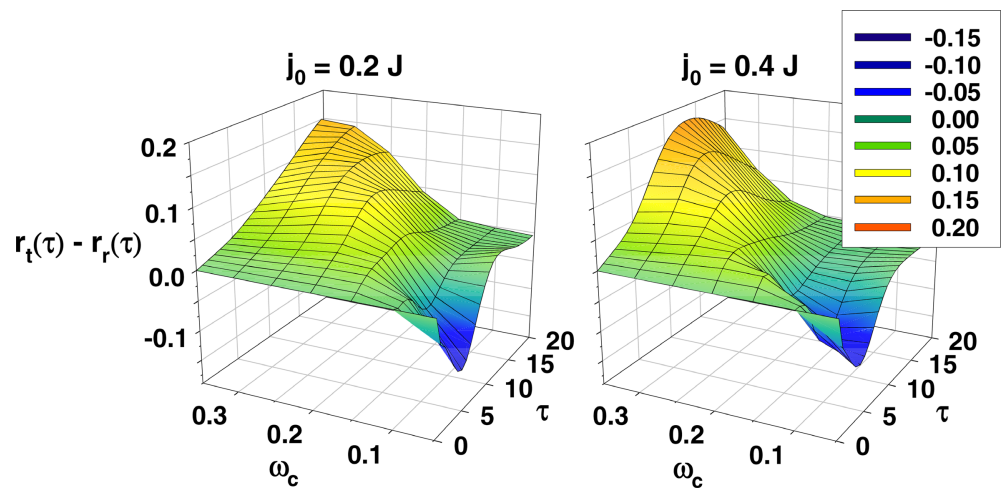

Fig. 5. The time and $\omega_{c}$ dependence of the difference between anisotropy of fluorescence $r_{t}-r_{r}$ of the LH2 (nonameric) ring and the LH4 (octameric) one for two strengths $j_{0}$ of dynamic disorder at temperature $T=0.5 \mathrm{~J}\left(j_{0}=0.2 \mathrm{~J}\right.$ - left, $j_{0}=0.4 \mathrm{~J}$ - right $)$

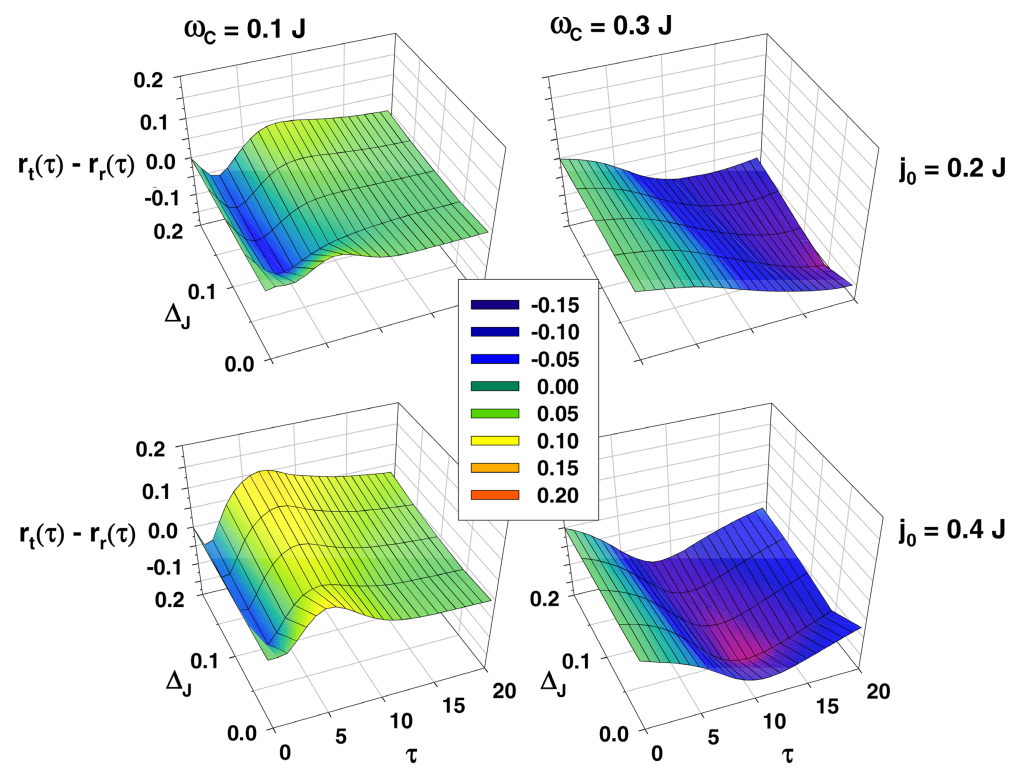

Fig. 6. The time and $\Delta_{J}$ dependence of the difference between the anisotropy of fluorescence $r_{t}-r_{r}$ of the LH2 (nonameric) ring and LH4 (octameric) one. The dynamic disorder is also included at $T=0.5 \mathrm{~J}\left(j_{0}=0.2 \mathrm{~J}\right.$ - upper row, $j_{0}=0.4 \mathrm{~J}$ - lower row, $\omega_{c}=0.1 J$ - left column, $\omega_{c}=0.3 J$ - right column).

Let us look at time decay of the anisotropy of fluorescence in the molecular ring with radial arrangement $r_{r}(t)$ - LH4, octameric one. Recently we discussed [19] results in the case of one type of the static disorder - uncorrelated Gaussian disorder in the local energies. 
In the present paper we concentrate on other type of the static disorder, the Gaussian uncorrelated disorder in the transfer integrals $J$, characterized by $\Delta_{J}$.

In Fig. 1. there is the time dependence of the anisotropy of fluorescence in case of the pure static disorder in transfer integrals for different $\Delta_{J}$. It is seen that the pure static disorder $\Delta_{J}=0.2$ leads to decay of the anisotropy of fluorescence from 0.7 to 0.4 within $\tau=20$.

Influence of the pure dynamic disorder, for two its strengths $j_{0}$ and different maxima $2 \omega_{c}$ of the spectral density function $J(\omega)$ is shown in Fig. 2. Dynamic disorder has a pronounced effect mainly in case of lower $\omega_{c}$.

Consequences of the combined static and dynamic disorder are presented in Fig.3. The time dependence of the anisotropy of fluorescence is displayed on four 3D graphs for two strengths of interaction with the bath and two maxima $2 \omega_{c}$ of the spectral density function. While for $\omega_{c}=0.1 J$ the influence of the static disorder is secondary due to dominance of the dynamic disorder, for larger $\omega_{c}=0.3 J$ the influence of the static disorder is more pronounced.

Comparison of the time dependence of fluorescence anisotropy for the octameric ring with radial arrangement of optical transition dipoles (like in LH4) and for the nonameric one with tangential arrangement of optical transition dipoles is presented graphically as differences $r_{t}(\tau)-r_{r}(\tau)$ in Figs 4-6.

\section{Conclusions}

The difference of the anisotropy of fluorescence between the nonameric tangentially arranged $\operatorname{ring} r_{t}(\tau)$ and octameric radially arranged one $r_{r}(\tau)$ for the static disorder in transfer integrals (Fig. 4) has an opposite sign in comparison with the result for the static disorder in local energies as shown in Fig. 2 in 19.

For the influence of the dynamic disorder (interaction with the bath), given by Eqs (911), we can conclude (Fig. 5) that the same differences are shifted to a smaller times for the stronger interaction $j_{0}$.

Similar conclusion can be drawn for the case of simultaneously acting static and dynamic disorder (shown in Fig. 3 and 6). We can also see more rapid decay of the anisotropy of fluorescence due to dynamic disorder in nonameric ring for smaller values of $\omega_{c}\left(\omega_{c}=0.1 \mathrm{~J}\right)$ (negative difference) and in octameric ring for larger values of $\omega_{c}\left(\omega_{c}=0.3 J\right)$ in presence of static disorder in transfer integrals (Fig. 6).

\section{Acknowledgement}

Support from the Ministry of Education, Youth and Sports of the Czech Republic (projects MSM0021620835 - I.B. and LC06002 - P.H.) is gratefully acknowledged.

\section{References}

1. de Ruijter, P.F., et al.: Biophysical J. 87, 3413 (2004)

2. Jang, S., Dempster, S.F., Silbey, R.J.: J. Phys. Chem. B 105, 6655 (2001) 
3. Sundström, V., Pullerits, T., van Grondelle, R.: J. Phys. Chem. B 103, 2327 (1999)

4. Novoderezhkin, V., van Grondelle, R.: J. Phys. Chem. B 106, 6025 (2002)

5. Nagarjan, V., Alden, R.G., Williams, J.C., Parson, W.W.: Proc. Natl. Acad. Sci. USA. 93, 13774 (1996)

6. Nagarjan, V., Johnson, E.T., Williams, J.C., Parson, W.W.: J. Phys. Chem. B 103, 2297 (1999)

7. Nagarjan, V., Parson, W.W.: J. Phys. Chem. B 104, 4010 (2000)

8. Rahman, T.S., Knox, R.S., Kenkre, V.M.: Chem. Phys. 44, 197 (1979)

9. Wynne, K., Hochstrasser, R.M.: Chem. Phys. 171, 179 (1993)

10. Kühn, O., Sundström, V., Pullerits, T.: Chem. Phys. 275, 15 (2002)

11. Heřman, P., Kleinekathöfer, U., Barvík, I., Schreiber, M.: J. Lumin. 447, 94-95 (2001)

12. Heřman, P., Kleinekathöfer, U., Barvík, I., Schreiber, M.: Chem. Phys. 275, 1 (2002)

13. Barvík, I., Kondov, I., Heřman, P., Schreiber, M., Kleinekathöfer, U.: Nonlin. Opt. 29, 167 (2002)

14. Heřman, P., Barvík, I.: Czech. J. Phys. 53, 579 (2003)

15. Reiter, M., Heřman, P., Barvík, I.: J. Lumin. 110, 258 (2004)

16. Heřman, P., Barvík, I., Reiter, M.: J. Lumin. 112, 469 (2005)

17. Heřman, P., Barvík, I.: J. Lumin. 558, 122-123 (2007)

18. Kumble, R., Hochstrasser, R.: J. Chem. Phys. 109, 855 (1998)

19. Heřman, P., Barvík, I., Zapletal, D.: J. Lumin. 128, 768 (2008)

20. Č́pek, V.: Z. Phys. B 99, 261 (1996)

21. Barvík, I., Macek, J.: J. Chin. Chem. Soc. 47, 647 (2000)

22. May, V., Kühn, O.: Charge and Energy Transfer in Molecular Systems. WileyWCH, Berlin (2000)

23. Pollard, W.T., Friesner, R.A.: J. Chem. Phys. 100, 5054 (1994)

24. Kondov, I., Kleinekathöfer, U., Schreiber, M.: J. Chem. Phys. 114, 1497 (2001) 